\title{
Does a coupling capacitor enhance the charge balance during neural stimulation? An empirical study
}

\author{
Marijn N. van Dongen ${ }^{1} \cdot$ Wouter A. Serdijn ${ }^{1}$
}

Received: 14 August 2014 / Accepted: 4 May 2015 / Published online: 29 May 2015

(C) The Author(s) 2015. This article is published with open access at Springerlink.com

\begin{abstract}
Due to their DC-blocking characteristic, coupling capacitors are widely used to prevent potentially harmful charge buildup at the electrode-tissue interface. Although the capacitors can be an effective safety measure, it often seems overlooked that coupling capacitors actually introduce an offset voltage over the electrode-tissue interface as well. This work investigates this offset voltage both analytically and experimentally. The calculations as well as the experiments using bipolar-driven platinum electrodes in a saline solution confirm that coupling capacitors introduce an offset, while they barely contribute to the passive charge balancing. In particular cases, this offset is shown to reach potentially dangerous voltage levels that could induce irreversible electrochemical reactions. This work therefore suggests that when the use of coupling capacitors is required, the offset voltage should be analyzed for all operating conditions to ensure it remains within safe boundaries.
\end{abstract}

Keywords Electric stimulation · Implantable neurostimulators $\cdot$ Electrodes

\section{Introduction}

Neural stimulation is becoming an increasingly popular clinical treatment methodology for a wide variety of diseases.

Marijn N. van Dongen

marijn.v.dongen@ieee.org

Wouter A. Serdijn

W.A.Serdijn@tudelft.nl

1 Section Bio-Electronics, Delft University of Technology, Mekelweg 4, 2628CD Delft, The Netherlands
In most cases, an implantable pulse generator (IPG) is used to deliver stimulation pulses to electrodes that are placed in the target area. The safety of the device is of major concern, since a faulty stimulation signal can cause irreversible damage to the neural tissue. It is especially important to prevent the flow of DCs through the electrodes [3, 7].

The use of coupling capacitors between the stimulator and the electrodes is widely considered to be an effective safety mechanism [10, 11], and indeed, various advantages concerning the use of coupling capacitors have been identified [4]. The first important advantage is the prevention of DCs in the event of device failure [9]. If, for example, one of the electrodes shorts to the supply voltage, the coupling capacitor will prevent a prolonged DC current through the electrodes.

The second important advantage that is attributed to coupling capacitors is that they improve the performance of passive charge-balancing techniques $[4,14,15]$. Charge balancing is important for polarizable electrodes to keep the electrode-tissue interface within an electrochemically safe regime [7]. A coupling capacitor helps due to its highpass characteristics, which limits the flow of DCs, and hence, no net charge can be injected into the tissue.

A disadvantage of coupling capacitors is that their required value is often too high to be integrated on an IC [15], and hence, they are realized using bulky external components. Many studies have focused on designing stimulator output stages with accurate charge-balancing circuits [8, 13] in order to eliminate the need of coupling capacitors. Others have proposed high-frequency operation to reduce their size [5]. Indeed, the results seem to suggest that the proposed mechanisms are good enough to prevent charge accumulation on the tissue even without coupling capacitors. However, it is not clear how these systems can guarantee safety in the event of a device failure. For this reason, many stimulator systems still require the use of coupling capacitors. 


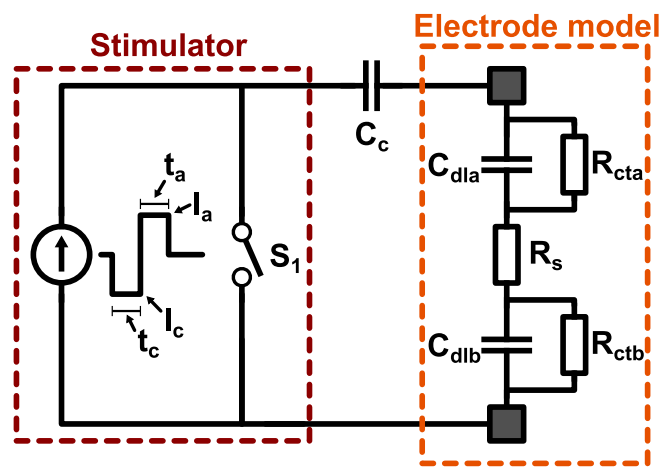

(a)

Fig. 1 a A basic setup of a biphasic constant current stimulator system is shown that includes a coupling capacitor $C_{\mathrm{c}}$ and an electrode model. $\mathbf{b}$ A picture of the measurement setup is shown with a detail

Although widely used, it often seems overlooked that a coupling capacitor eliminates control over the DC voltage across the electrodes. As will be shown in this work, it is therefore possible for an offset voltage $V_{\text {os }}$ to develop over the electrode-tissue interface, even when the electrodes and capacitors are shorted in between the stimulation pulses and charge-balanced biphasic stimulation is used.

If $V_{\text {os }}$ becomes too large, the electrode-tissue interface may leave the electrochemically safe regime, triggering the production of potentially dangerous reaction products. In this case, the intended safety mechanisms of the coupling capacitor create the opposite result: A potentially dangerous situation is created. In this work, the value of $V_{\text {os }}$ is analyzed over various operating conditions, both analytically and experimentally. This gives insight in when $V_{\text {os }}$ is exceeding a predefined safe regime.

\section{Methods}

A basic setup of a biphasic stimulator system is depicted in Fig. 1a: The coupling capacitor $C_{\mathrm{c}}$ is connected in series with the stimulator and the electrodes. The stimulation source in Fig. 1a is a biphasic constant current stimulator with a cathodic first stimulation pulse with amplitude $I_{\mathrm{c}}$ and duration $t_{\mathrm{c}}$. The anodic charge cancelation phase follows with amplitude $I_{\mathrm{a}}$ and duration $t_{\mathrm{a}}$. Most stimulator systems apply a passive charge-balancing scheme [15], in which the series connection of the electrodes and coupling capacitor are shorted after the stimulation cycle by closing switch $S_{1}$ to discharge $C_{\mathrm{dl}}$. The duration of shorting $t_{\mathrm{dis}}$ is determined by the repetition rate $f_{\text {stim }}=1 / t_{\text {stim }}$ of the stimulation, since $S_{1}$ needs to be opened again when the next stimulation cycle starts.

As shown in Fig. 1a, the electrodes are modeled as a resistance $R_{\mathrm{s}}$ in series with capacitors $\left(C_{\mathrm{dla}}\right.$ and $\left.C_{\mathrm{dlb}}\right)$ and

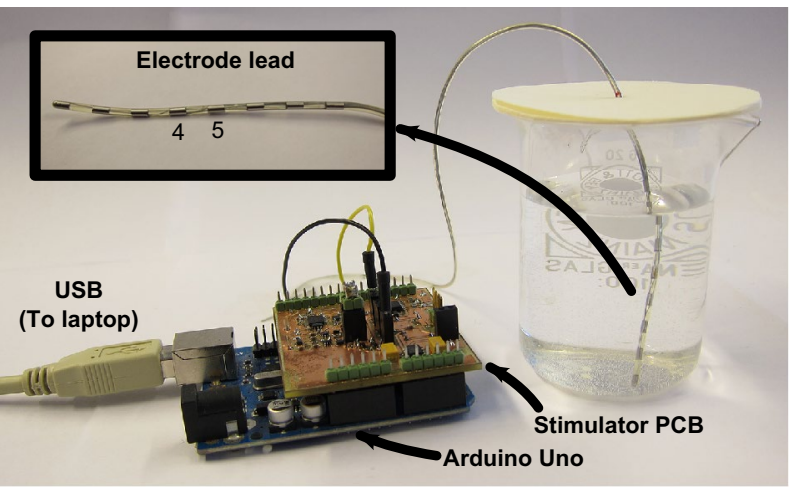

(b)

of the electrode lead where contacts 4 and 5 , which were used for stimulation, are indicated

resistors $\left(R_{\mathrm{cta}}\right.$ and $\left.R_{\mathrm{ctb}}\right)$ that model the electrode-tissue interfaces of both electrodes [6]. The electrodes used in this study are single percutaneous octrode leads (manufactured by ANS, currently St. Jude Medical): They consist of eight ring-shaped platinum contacts that are distributed on a single lead. Each electrode has a diameter of $1.5 \mathrm{~mm}$ and a width of $3 \mathrm{~mm}$ (area $0.14 \mathrm{~cm}^{2}$ ). A picture of the electrodes is depicted in Fig. 1b. These types of electrodes are typically used for spinal cord stimulation, and the stimulation amplitudes used in this paper are based on the specifications of the EONTM IPG (also from St. Jude Medical) [16]. The electrodes were submerged in a phosphate-buffered saline (PBS) solution containing the following: $1.059 \mathrm{mM}$ $\mathrm{KH}_{2} \mathrm{PO}_{4}, 155.172 \mathrm{mM} \mathrm{NaCl}, 2.966 \mathrm{mM} \mathrm{Na} 2 \mathrm{HPO}_{4}-7 \mathrm{H}_{2} \mathrm{O}$ (pH 7.4, Gibco ${ }^{\circledR}$ Life technologies ${ }^{\mathrm{TM}}$ ). The electrodes were connected in a bipolar fashion by selecting contacts 4 and 5 as the anode and cathode (see Fig. 1b). The other contacts were left floating.

Using an HP4194A impedance analyzer (excitation amplitude $0.1 \mathrm{~V}$ ), it was found that for these electrodes in the PBS solution, $R_{\mathrm{s}} \approx 100 \Omega$ and $C_{\mathrm{dl}} \approx 1.5 \mu \mathrm{F}$. Here, $C_{\mathrm{dl}}$ is the capacitive part of both electrode-tissue interfaces combined. The value of $R_{\mathrm{ct}} \approx 1 \mathrm{M} \Omega$ (also combining both interfaces) was determined by measuring the voltage over the electrodes due to a 5-nA DC from a Keithley 6430 subfemtoamp sourcemeter.

\subsection{Determining $V_{\text {os }}$}

After the anodic phase, both $C_{\mathrm{c}}$ and $C_{\mathrm{dl}}$ will be charged. Upon closing $S_{1}$, these capacitors will be discharged with a time constant:

$\tau_{\mathrm{dis}}=R_{s} C_{\mathrm{eq}} \quad C_{\mathrm{eq}}=\frac{C_{c} C_{\mathrm{dl}}}{C_{c}+C_{\mathrm{dl}}}$ 


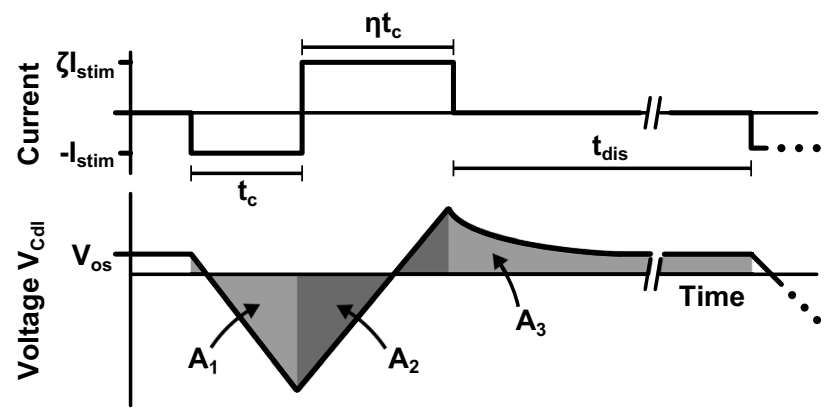

Fig. 2 Schematic plot of $V_{\mathrm{Cdl}}$ during a biphasic stimulation cycle with charge mismatch. When $V_{\text {os }}$ is stable, the area $A_{1}+A_{2}+A_{3}$ equals zero

If $S_{1}$ would be closed sufficiently long, a pseudosteady state is reached in which:

$V_{\mathrm{Cc}}+V_{\mathrm{Cdl}}=0$

Here, $V_{\mathrm{Cc}}$ is the voltage over $C_{\mathrm{c}}$. If $S_{1}$ is closed even longer, $C_{\mathrm{dl}}$ will continue to discharge through $R_{\mathrm{ct}}$ with time constant $\tau_{2}=R_{\mathrm{ct}} C_{\mathrm{dl}}$ until $V_{\mathrm{Cdl}}=0 \mathrm{~V}$ and the actual steady state is reached. However, usually $t_{\text {dis }} \ll \tau_{2}$, and therefore, only the pseudosteady state is reached.

Note that Eq. (2) does not guarantee that $V_{\mathrm{Cdl}}=0$ in pseudosteady state: It is an under-determined equation, and $V_{\mathrm{Cc}}=-V_{\mathrm{Cdl}}$ can have any value. Only when both $C_{\mathrm{c}}$ and $C_{\mathrm{dl}}$ are ideal capacitors, the same current is flowing through both capacitors during a stimulation cycle, which causes $V_{\mathrm{Cc}}=V_{\mathrm{Cdl}}=0 \mathrm{~V}$ in pseudosteady state. If these requirements are not met (e.g., when $R_{\mathrm{ct}} \neq \infty$ ), the current though $C_{\mathrm{c}}$ does not equal to the current through $C_{\mathrm{dl}}$, which will cause $V_{\mathrm{Cdl}}=-V_{\mathrm{Cc}} \neq 0$ in pseudosteady state. This charge imbalance can accumulate over many stimulation cycles, which creates an offset in $V_{\mathrm{Cdl}}$ [2].

We refer to Fig. 2 to analyze $V_{\mathrm{Cdl}}$ when after many stimulation cycles the offset voltage $V_{\mathrm{os}}$ is stable. In order for this voltage to be stable, the average current through $R_{\mathrm{ct}}$ must be zero such that no charge is lost that causes an inequality in the charge accumulated on $C_{\mathrm{dl}}$ with respect to $C_{c}$. Therefore, it must hold that the average value of $V_{\mathrm{Cdl}}$ (and hence the area as indicated in Fig. 2) is zero as well.

To find the value of $V_{\text {os }}$ for which this requirement is met, it is assumed that the cathodic stimulation phase is characterized by a duration $t_{\mathrm{c}}$ and amplitude $I_{\mathrm{c}}=I_{\text {stim. }}$. In the anodic phase, both the duration $t_{\mathrm{a}}=\eta t_{\mathrm{c}}$ and the amplitude $I_{\mathrm{a}}=\zeta I_{\text {stim }}$ can include mismatch. Furthermore, it is assumed that $t_{\text {dis }} \ll \tau_{2}$ (such that pseudosteady state is reached) and that $R_{\mathrm{ct}}$ is large enough to be neglected in the analysis (but as stated above, it must be finite). The areas $A_{1}, A_{2}$ and $A_{3}$ are found as:

$A_{1}=\int_{0}^{t_{\mathrm{c}}}\left(V_{\mathrm{os}}-\frac{I_{\mathrm{stim}} t}{C_{\mathrm{dl}}}\right) \mathrm{d} t=V_{\mathrm{os}} t_{\mathrm{c}}-\frac{I_{\mathrm{stim}} t_{\mathrm{c}}^{2}}{2 C_{\mathrm{dl}}}$

$$
\begin{aligned}
A_{2} & =\int_{0}^{\eta t_{\mathrm{c}}}\left(V_{\mathrm{os}}-\frac{I_{\mathrm{stim}} t_{\mathrm{c}}}{C_{\mathrm{dl}}}+\frac{\zeta I_{\mathrm{stim}} t}{C_{\mathrm{dl}}}\right) \mathrm{d} t \\
& =V_{\mathrm{os}} \eta t_{\mathrm{c}}-\frac{I_{\mathrm{stim}} \eta t_{\mathrm{c}}^{2}}{C_{\mathrm{dl}}}+\frac{\zeta I_{\mathrm{stim}}\left(\eta t_{\mathrm{c}}\right)^{2}}{2 C_{\mathrm{dl}}} \\
A_{3} & =\int_{0}^{t_{\mathrm{dis}}}\left(V_{\mathrm{os}}-(1-\zeta \eta) \frac{I_{\mathrm{stim}} t_{\mathrm{c}}}{C_{\mathrm{dl}}} \exp \left(\frac{-t}{R_{\mathrm{s}} C_{\mathrm{dl}}}\right)\right) \mathrm{d} t \\
& =V_{\mathrm{os}} t_{\mathrm{dis}}-(1-\zeta \eta) I_{\mathrm{stim}} t_{\mathrm{c}} R_{\mathrm{s}}
\end{aligned}
$$

By setting $A_{1}+A_{2}+A_{3}=0$ and solving for $V_{\text {os }}$, the following equation is obtained:

$V_{\mathrm{os}}=\frac{\left(0.5+\eta-0.5 \zeta \eta^{2}\right) I_{\mathrm{stim}} t_{\mathrm{c}}^{2}+(1-\zeta \eta) I_{\mathrm{stim}} C_{\mathrm{dl}} t_{\mathrm{c}}}{C_{\mathrm{dl}} t_{\mathrm{c}}(1+\eta)+t_{\mathrm{dis}}}$

If $\zeta=\eta=1$, which means that perfectly charge-balanced stimulation is applied, the following equation holds:

$V_{\mathrm{os}}=\frac{I_{\mathrm{stim}} t_{\mathrm{c}}^{2}}{C_{\mathrm{dl}}\left(2 t_{\mathrm{c}}+t_{\mathrm{dis}}\right)}=\frac{I_{\mathrm{stim}} t_{\mathrm{c}}^{2}}{C_{\mathrm{dl}}\left(t_{\mathrm{stim}}\right)}$

In Fig. 3a, the value of $V_{\text {os }}$ is depicted for a chargebalanced stimulation cycle with $f_{\text {stim }}=200 \mathrm{~Hz}$. This cycle includes a coupling capacitor. For small $I_{\text {stim }}$ and $t_{\mathrm{c}}$, the value of $V_{\text {os }}$ is small and will have negligible influence on the system. However, for larger stimulation intensities, $V_{\text {os }}$ starts to increase toward several hundreds of millivolts (up to $800 \mathrm{mV}$ for the maximum intensity).

Equation (4) can also be used to analyze monophasic stimulation patterns by choosing $\eta=0$. In Fig. 3b, the values of $V_{\text {os }}$ are plotted for this situation. Somewhat surprisingly, these values are smaller than the biphasic charge-balanced stimulation. However, this can be explained by the fact that due to the relatively low value of $R_{\mathrm{s}}$, the discharge current during $t_{\mathrm{dis}}$ is larger than $I_{\text {stim }}$, and hence, the electrodes discharge faster toward pseudosteady state as compared to the biphasic stimulation waveform.

\subsection{Verifying $V_{\text {os }}$}

To verify Eq. (4), the response of an electrode system was analyzed using both simulations and measurements in a saline bath. To simulate the response of these electrodes, the circuit from Fig. 1 was implemented in a simulator (LT-Spice). Switch $S_{1}$ was chosen to have $R_{\text {off }}=10 \mathrm{M} \Omega$ to mimic the limited output impedance of the current source and $R_{\mathrm{on}}=10 \Omega$. The stimulation current was chosen to be $I_{\text {stim }}=1.5 \mathrm{~mA}(\zeta=1)$, while an $8 \%$ charge mismatch was introduced by making $t_{\mathrm{c}}=460 \mu \mathrm{s}$ and $t_{\mathrm{a}}=500 \mu \mathrm{s}$ $(\eta=1.087)$. After the stimulation cycle, switch $S_{1}$ was closed for $t_{\text {dis }}=9 \mathrm{~ms}$ before the next stimulation pulse is started. This makes the stimulation repetition rate slightly higher than $100 \mathrm{~Hz}$. 


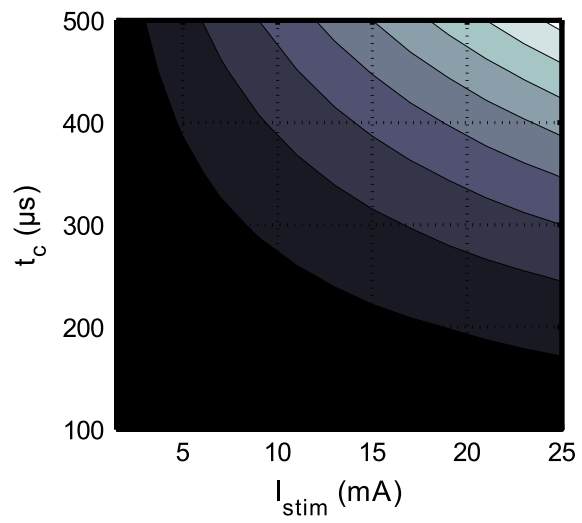

(a)

Fig. 3 Overview of the pseudosteady-state offset voltages $V_{\text {os }}$ for a variety of stimulation settings. a A perfectly charge-balanced stimulation waveform is chosen, and $V_{o s}$ is determined according to Eq. (5)

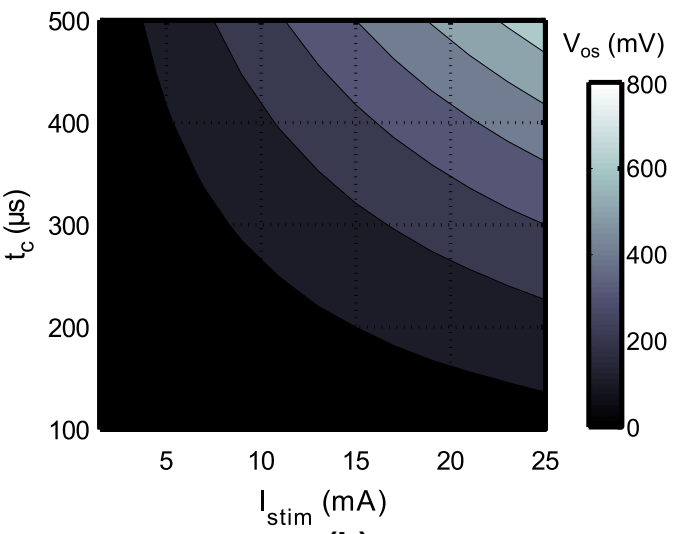

(b)

with $f_{\text {stim }}=200 \mathrm{~Hz}$. b A monophasic stimulation waveform is used, and $V_{\text {os }}$ is determined using Eq. (4) with $\eta=0$

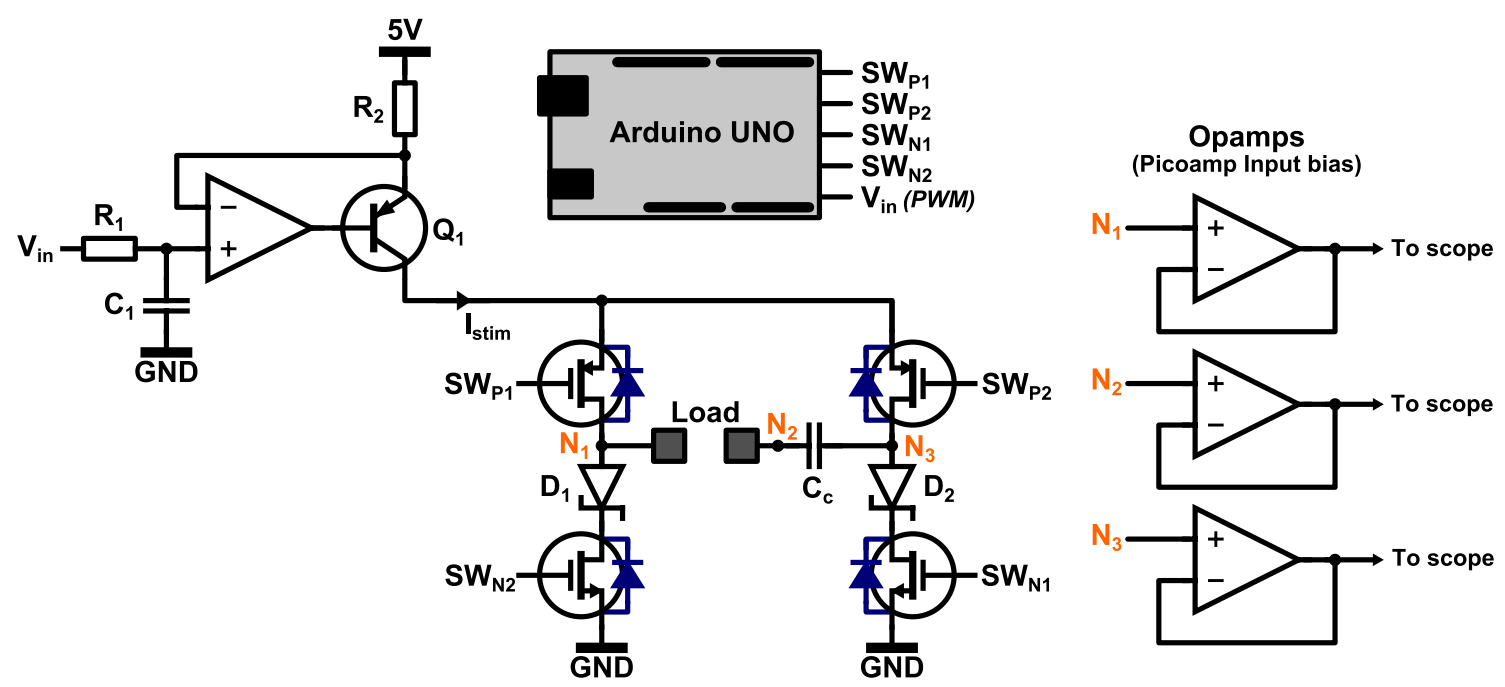

Fig. 4 Measurement setup used to verify the influence of the coupling capacitor $C_{c}$ on the charge cancelation. A constant current source implemented using $Q_{1}$ is connected to the load via an H-bridge configuration (MOSFET switches), which allows bidirec-

Using Eq. (1), it is found that $t_{\text {dis }}>60 \tau_{\text {dis }}$, which means that $V_{\mathrm{Cdl}}$ and $V_{\mathrm{Cc}}$ can be assumed to have reached their pseudosteady-state values. Also $\tau_{2}=1.5 \mathrm{~s} \gg \tau_{\text {dis }}$, which means that the system will stay in pseudosteady state and will not have the opportunity to fully discharge.

The value of $C_{\mathrm{c}}$ should be chosen well above $C_{\mathrm{dl}}$ in order to limit the contribution of $C_{\mathrm{c}}$ to the voltage headroom of the stimulator [15]. In this particular case, it was chosen to make $C_{\mathrm{c}}=8.8 \mu \mathrm{F}$, based on the availability of components for the measurements. The circuit was simulated over many stimulation cycles (up to $200 \mathrm{~s}$ ) to analyze the voltage over $C_{\mathrm{dl}}$ and $C_{\mathrm{c}}$. To minimize leakage introduced by the simulation setup, the minimum conductance of tional stimulation. An Arduino Uno is used for the control of the circuit, while buffers are used to prevent loading of the system during measurements

the SPICE simulator was lowered from $G_{\min }=1 \mathrm{p} \Omega^{-1}$ to $G_{\min }=1 \mathrm{f} \Omega^{-1}$. After a simulation, MATLAB was used to select the time stamps that correspond to pseudosteady state to obtain the values of $V_{\text {os }}$ over many stimulation cycles.

After simulations, a stimulation circuit was built using discrete components as depicted in Fig. 4. Transistor $Q_{1}$ (2N3906) implements a current source together with resistor $R_{2}$ and the opamp (LMV358). The output current $I_{\text {stim }}$ is controlled using the PWM signal $V_{\text {in }}$ that is filtered using $R_{1}=1 \mathrm{M} \Omega$ and $C_{1}=1 \mu \mathrm{F}$. Using the H-bridge topology implemented with MOSFET devices (NTZD3155C), the current can be injected bidirectionally through the load during the cathodic and anodic stimulation phase. An Arduino 
Table 1 Stimulation settings used during measurements

\begin{tabular}{lllllll}
\hline Nr. & Waveform & $I_{\text {stim }}(\mathrm{mA})$ & $t_{\mathrm{c}}(\mu \mathrm{s})$ & Mismatch $\eta$ & $f_{\text {stim }}(\mathrm{Hz})$ & Incl. $C_{\mathrm{c}}$ ? \\
\hline 1 & Biphasic & 1.5 & 460 & $1.085\left(t_{\mathrm{a}}=500 \mu \mathrm{s}\right)$ & 110 & No \\
2 & Biphasic & 1.5 & 460 & $1.085\left(t_{a}=500 \mu \mathrm{s}\right)$ & 110 & Yes \\
3 & Biphasic & 15 & 200 & $0.75\left(t_{a}=150 \mu \mathrm{s}\right)$ & 400 & Yes \\
4 & Monophasic & 15 & 200 & 0 & 100 & Yes \\
\hline
\end{tabular}

Uno is used to control the switches: During the cathodic phase, switches $S W_{\mathrm{P} 1}$ and $S W_{\mathrm{N} 1}$ are closed, while during the anodic phase, switches $S W_{\mathrm{P} 2}$ and $S W_{\mathrm{N} 2}$ are closed. The tissue is shorted in between the stimulation pulses by closing $S W_{\mathrm{P} 1}$ and $S W_{\mathrm{P} 2}$. Diodes $D_{1}$ and $D_{2}$ (CD0603-B00340) are needed to prevent unwanted current flow through the body diodes of $S W_{\mathrm{N} 1}$ and $S W_{\mathrm{N} 2}$ : If $C_{\mathrm{dl}}$ is charged beyond $0.6 \mathrm{~V}$ during the cathodic phase, the body diodes of $S W_{\mathrm{N} 1}$ and $S W_{\mathrm{N} 2}$ otherwise become forward biased when the stimulation direction is reversed.

The Arduino was programmed with four different stimulation settings as summarized in Table 1. The first setup uses no coupling capacitor, and it is verified that $C_{\mathrm{dl}}$ is indeed charged back to $0 \mathrm{~V}$ after a stimulation cycle. The second setup uses a low-intensity stimulation cycle with a positive charge mismatch, while the third setup uses a highintensity stimulation cycle (close to the maximum stimulation intensity possible before the current source would clip to the $5 \mathrm{~V}$ supply voltage). The fourth experiment uses a monophasic stimulation waveform. All measurements were taken after stimulation was enabled sufficiently long (at least $5 \mathrm{~min}$ ) to allow the voltages to settle.

The load of the circuit in Fig. 4 first consisted of the electrode model from Fig. $1\left(R_{\mathrm{s}}=100 \Omega, C_{\mathrm{dl}}=1.5 \mu \mathrm{F}\right.$, $R_{\text {ct }}=1 \mathrm{M} \Omega$ ). Subsequently, the electrode model was replaced by the electrodes that were submerged in the PBS solution.

To measure the response of the system, the relevant output signals are buffered using picoampere input bias operational amplifiers (AD8625, powered with $\pm 8 \mathrm{~V}$ ) in order to prevent the measurement equipment from loading the system. It was found using simulations and measurements that a $10 \mathrm{M} \Omega \| 12 \mathrm{pF}$ standard probe largely distorts the measurement, as will be discussed further in the last section.

\section{Results}

Figure 5 shows the simulation results of the circuit from Fig. 1. In Fig. 5a, the value of $V_{\mathrm{Cdl}}$ in pseudosteady state is shown over many stimulation cycles. When no coupling capacitor is used, $V_{\mathrm{Cdl}}$ can discharge almost completely. When $C_{\mathrm{c}}$ is added in Fig. 5a, it is seen that after several stimulation cycles, $V_{\mathrm{Cdl}}=20.7 \mathrm{mV}$. Indeed, the introduction of $C_{\mathrm{c}}$ causes an offset in $V_{\mathrm{Cdl}}$ in pseudosteady state.
Furthermore, the simulated values correspond well with Eq. 4, which predicts $V_{\text {os }}=20.6 \mathrm{mV}$.

In Fig. 5b, c, the simulated transient behavior of the voltages in the circuit including $C_{\mathrm{c}}$ is shown for two time instances. Figure $5 \mathrm{~b}$ shows the voltages right after the first stimulation cycle, while Fig. $5 c$ shows the voltages during a stimulation cycle after $190 \mathrm{~s}$ of simulation time, where the offset is clearly visible.

In Fig. 6, the measurement results are presented for all experiments listed in Table 1 . In all figures, $V_{\text {out }}$ refers to the voltage measured over the output of the current source (between nodes $N_{1}$ and $N_{3}$ in Fig. 3) and $V_{\text {el }}$ is the voltage over the electrode (nodes $N_{1}$ and $N_{2}$ ). For saline measurements, it is not possible to measure $V_{\mathrm{Cdl}}$ directly, and hence, $V_{\mathrm{el}}$ is shown instead.

\section{Discussion}

The measured values for $V_{\mathrm{os}}$ are summarized in Table 2 and compared with the values calculated using Eq. (4). It is seen that the measurements with the model correspond well to the calculated values, indicating that the circuit implementation is working as expected. For the saline measurements, the values of $V_{\mathrm{os}}$ are higher than expected, and hence, the model underestimates the offset value introduced. This is most likely due to complex nonlinear behavior of the electrode-tissue interface that cannot be modeled using the simple capacitance $C_{\mathrm{dl}}$. The electrode model is a small-signal model ( $C_{\mathrm{d}}$ was found using a sinusoidal excitation of $0.1 \mathrm{~V}$ ), and the measurement results show that the validity of the model is limited during a stimulation cycle. From these results and the plots in Fig. 6, we can draw three important conclusions.

1 First of all, coupling capacitors barely improve the way in which $V_{\mathrm{Cdl}}$ returns to equilibrium. The only way in which $C_{\mathrm{c}}$ contributes is by making $\tau_{\mathrm{dis}}$ (Eq. 1) smaller during the $t_{\mathrm{dis}}$ interval [4]. This causes the interface to discharge toward equilibrium slightly faster. However, since $C_{\mathrm{c}} \gg C_{\mathrm{dl}}$, the influence on $\tau_{\mathrm{dis}}$ is negligible, and hence, coupling capacitors barely improve the charge cancelation.

2 Second of all, coupling capacitors introduce an offset in the pseudosteady-state value of the electrodes. The 


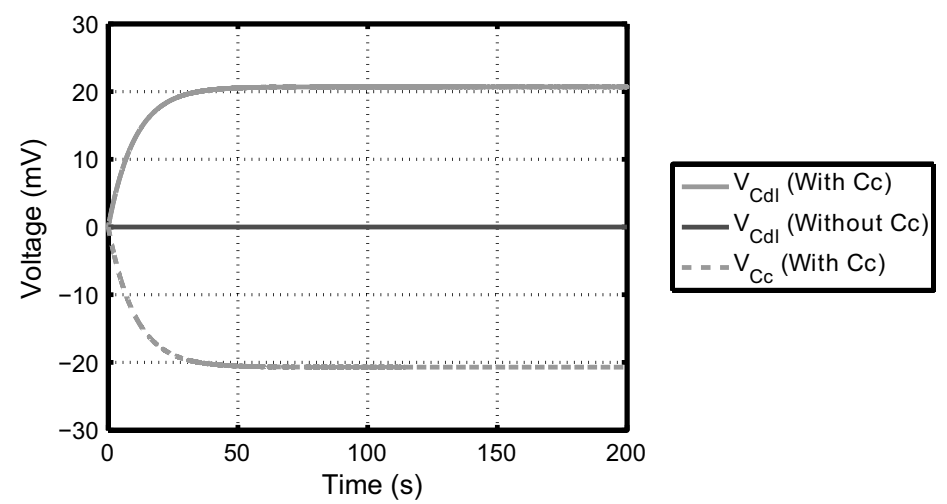

(a)

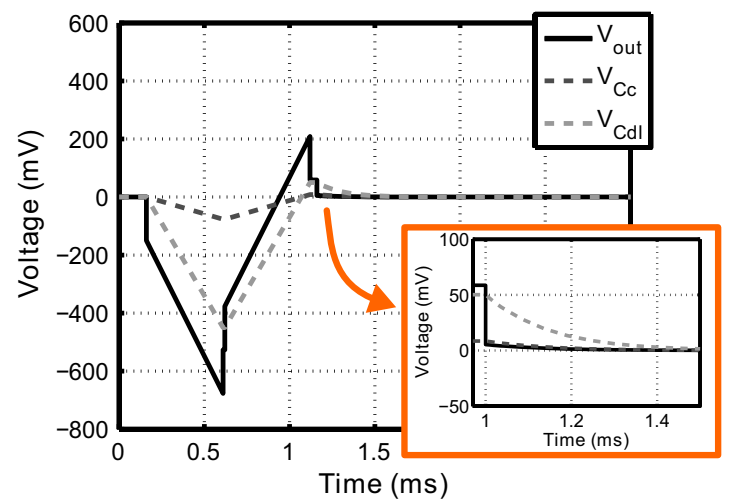

(b)

Fig. 5 Simulation results of the circuit from Fig. 1. a The voltages $V_{\mathrm{Cdl}}$ and $V_{\mathrm{Cc}}$ are shown during the interval $t_{\text {open }}$ over a large number of stimulation cycles. As can be seen, the coupling capacitor causes

value of $V_{\text {os }}$ can be predicted using Eq. (4), although it was found that this equation underestimated the offset measured from the electrodes in saline.

The question is whether or not $V_{\text {os }}$ introduces potential safety issues. For small values of $V_{\text {os }}$, no problems are likely to occur: As long as no irreversible faradaic reactions are triggered, no harmful effects are to be expected. Even more so, $V_{\text {os }}$ will increase the amount of charge that can be injected [1], because $V_{\text {os }}$ reduces the peak voltage of $V_{\mathrm{Cdl}}$ during a stimulation cycle.

However, when $V_{\text {os }}$ increases toward the threshold of irreversible faradaic reactions $(600-900 \mathrm{mV}$ for platinum electrodes [12]), problems can be expected. In this case, the interface is experiencing a significant offset voltage during the $t_{\text {dis }}$ interval, during which irreversible reactions might occur. For high stimulation intensities, Fig. 3 predicts values of $V_{\text {os }}$ that are close to or exceed the maximum safe voltage window.

3 Finally, secondary effects can have a strong effect on $V_{\text {os }}$. Using the settings of experiment 2, measurements

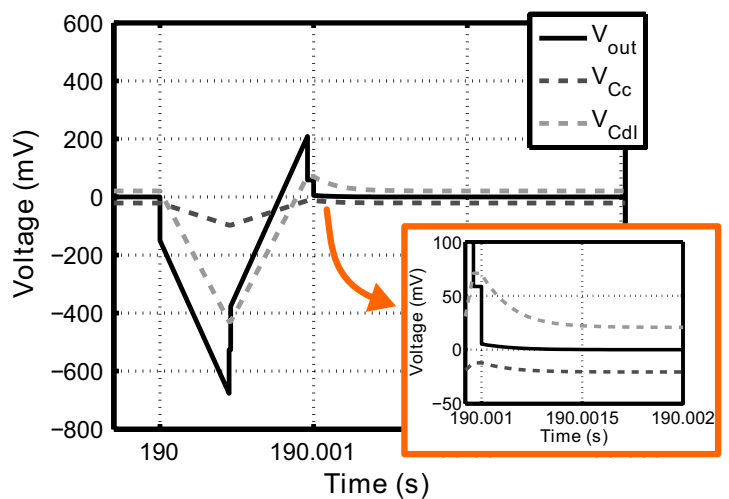

(c)

an offset. b, $\mathbf{c}$ The transient voltages are shown for the system with $C_{\mathrm{c}}$ and $R_{\mathrm{p}}=\infty$ just after stimulation is initiated and after $190 \mathrm{~s}$, respectively

were repeated with both the model and the electrodes as load. This time, the voltages were not buffered using the picoampere input bias opamps, but $10 \mathrm{M} \Omega \| 12 \mathrm{pF}$ probes (referenced to ground) were connected to $N_{1}, N_{2}$ and $N_{3}$ directly. This has a large impact on the offset voltage: It increases from $25 \mathrm{mV}$ to $2 \mathrm{~V}$ (model) and from $80 \mathrm{mV}$ to $0.6 \mathrm{~V}$ (saline).

All in all, it can be concluded that in contrast to what many other studies have suggested [4, 14, 15], the introduction of $C_{\mathrm{c}}$ does not improve the charge-balancing process and it is furthermore associated with the loss of control over the pseudosteady-state value of $V_{\mathrm{Cdl}}$. Instead of ensuring safety by returning the electrode interface voltage back to $0 \mathrm{~V}$, the coupling capacitor introduces an unwanted offset in the interface voltage that is hard to control by the stimulator and, moreover, is sensitive to secondary effects.

Although this work suggests that coupling capacitors are not beneficial for charge cancelation purposes, they still protect the electrodes and tissue from DCs in case of a device or software failure. Depending on the application, this could require the need to still use these capacitors. In 


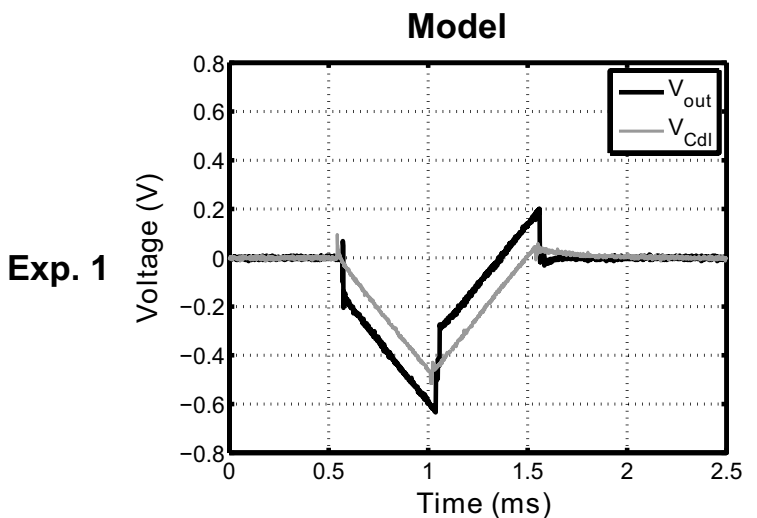

(a)

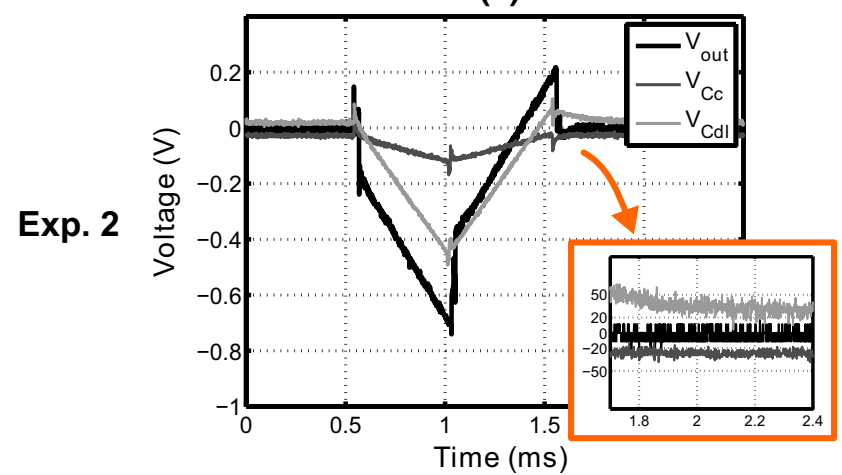

(c)

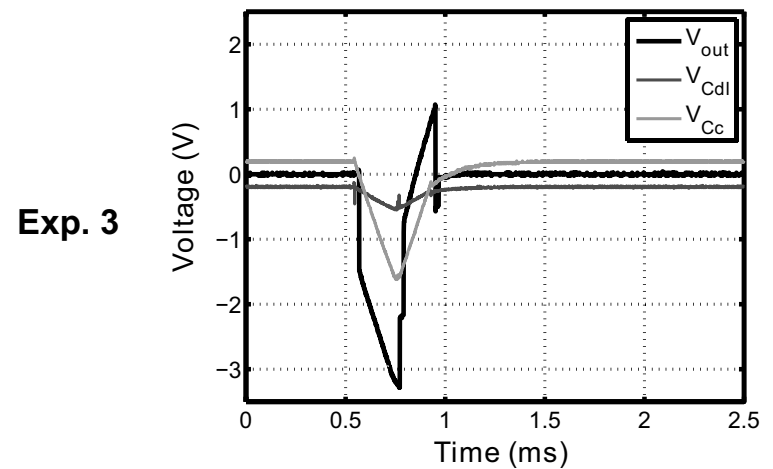

(e)

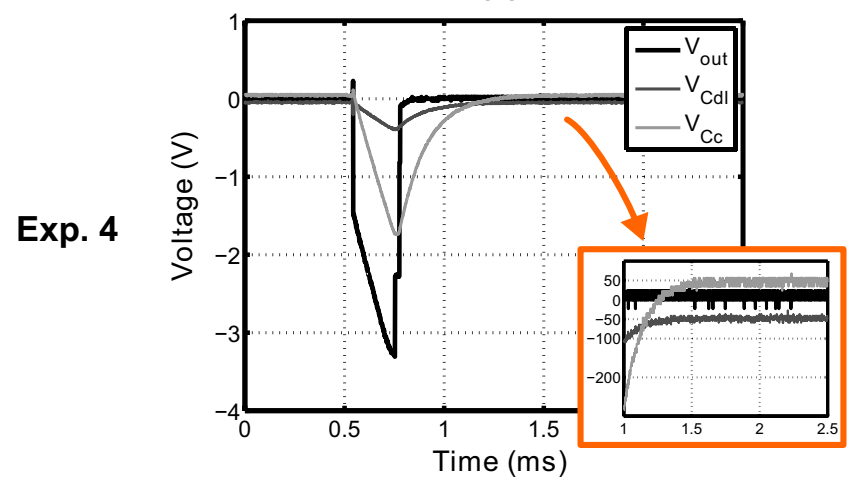

(g)

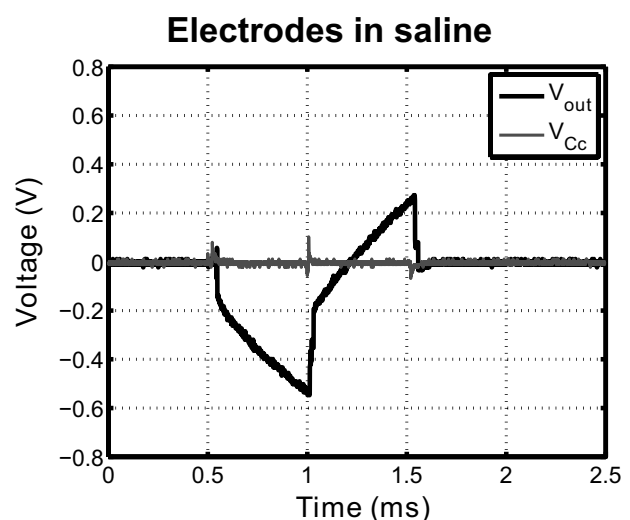

(b)

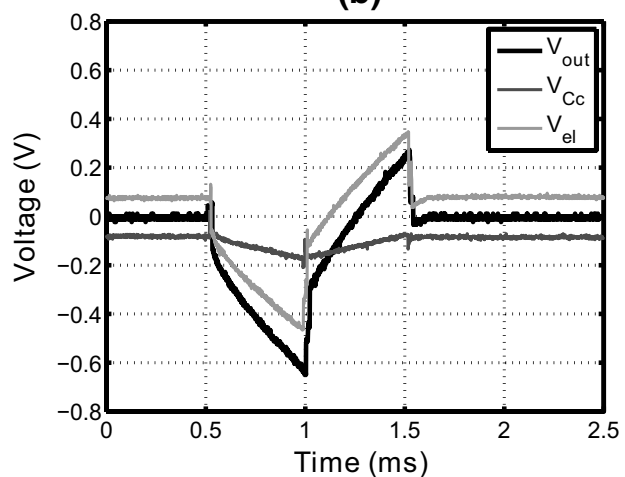

(d)

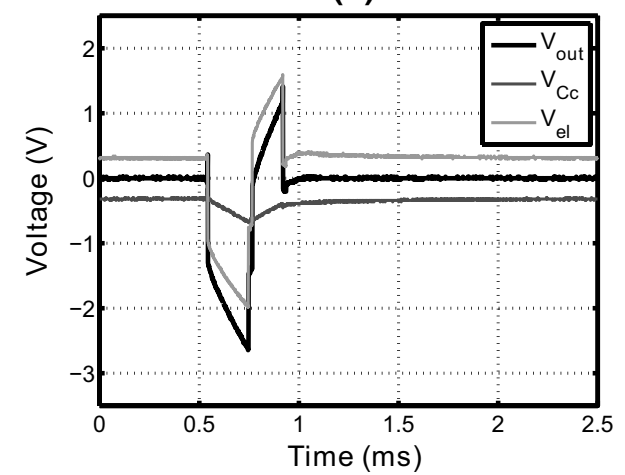

(f)

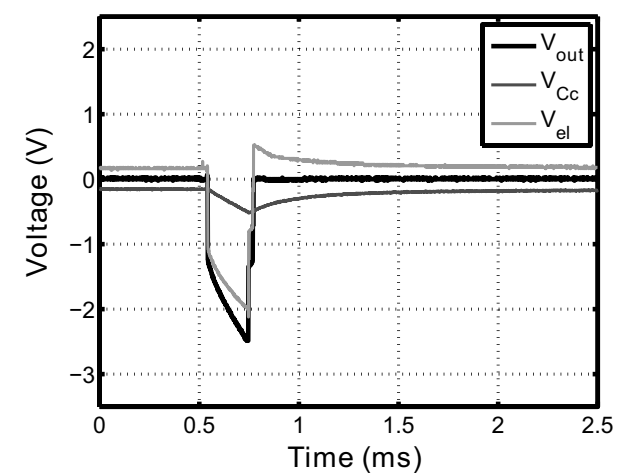

(h)
Fig. 6 Measurement results from the experimental setup depicted in Fig. 4 with the load consisting of the electrode model (left column) and the electrodes in saline (right column). For both loads, four stim- ulation settings are used as described in Table 1. As can be seen, the offset voltage depends on the stimulation settings used, but is zero when no coupling capacitor is used (experiment 1 ) 
Table 2 Calculated and measured values of $V_{\text {os }}$ for the experiments summarized in Table 1

\begin{tabular}{llcc}
\hline Experiment & Equation (4) $(\mathrm{mV}) \begin{array}{c}\text { Measurement } \\
(\text { model })(\mathrm{mV})\end{array}$ & $\begin{array}{l}\text { Measurement } \\
(\text { saline })(\mathrm{mV})\end{array}$ \\
\hline 1 & 0 & 0 & 0 \\
2 & 21.6 & 25 & 80 \\
3 & 201 & 200 & 320 \\
4 & 50 & 50 & 165 \\
\hline
\end{tabular}

that case, the results from this study show that the stimulation settings should be limited to ensure that under all operating conditions, $V_{\text {os }}$ does not exceed any predefined safety window.

It is possible to discharge both $C_{\mathrm{c}}$ and $C_{\mathrm{dl}}$ completely by introducing an additional switch over $C_{\mathrm{c}}$. In this case, $C_{\mathrm{c}}$ and $C_{\mathrm{dl}}$ are shorted individually and are guaranteed to discharge toward $0 \mathrm{~V}$ in pseudosteady state, which would eliminate the offset. However, in this case, it is unclear how the coupling capacitor is contributing to the chargebalancing process: It does not improve $\tau_{\text {dis }}$ and $V_{\mathrm{Cdl}}$ will have the same response as compared to the circuit without $C_{\mathrm{c}}$. Furthermore, the additional switch introduces a singlefault device failure risk. Therefore, the advantages of a coupling capacitor are not exploited when $C_{\mathrm{c}}$ is discharged separately.

This work focused on passive charge-balancing techniques. Active charge-balancing techniques use feedback to bring the electrode voltage back to safe values after a stimulation cycle [15] and can therefore help to overcome the offset problem. However, if these schemes require a coupling capacitor to protect in the event of a device failure, it is important to measure the voltage over the electrodes only and not to include the coupling capacitor. This requires an additional sensing pin if the coupling capacitors are realized using external components. Only then, the feedback mechanism will help to remove the offset.

In this study, only one type of electrode was considered. Smaller electrodes have different impedance levels, and more research is needed to find the pseudosteady-state response in this case. Note that Eq. (4) is only valid under the assumption that $\tau_{\mathrm{dis}} \gg t_{\mathrm{dis}}$, which might not be the case for high impedance electrodes. Finally, it would also be interesting to determine the influence of the coupling capacitors in vivo.

\section{Conclusions}

In this work, the influence of coupling capacitors on the charge-balancing properties is studied during neural stimulation. In contrast to what previous work suggests, coupling capacitors were found not to improve the chargebalancing process. Even more so, they introduce an offset voltage in the electrodes, which cannot be removed by conventional means such as passive discharging. The value of the offset voltage depends on the stimulation and electrode parameters. When using coupling capacitors, it is therefore important to ensure that this offset voltage does not exceed any safety boundaries for all possible operating conditions.

Open Access This article is distributed under the terms of the Creative Commons Attribution 4.0 International License (http://creativecommons.org/licenses/by/4.0/), which permits unrestricted use, distribution, and reproduction in any medium, provided you give appropriate credit to the original author(s) and the source, provide a link to the Creative Commons license, and indicate if changes were made.

\section{References}

1. de Donaldson NN, Donaldson PEK (1986) When are actively balanced biphasic ('Lilly') stimulating pulses necessary in a neurological prosthesis? II $\mathrm{pH}$ changes; noxious products; electrode corrosion; discussion. Med Biol Eng Comput 24(1):50-56

2. de Donaldson NN, Donaldson PEK (1986) When are actively balanced biphasic ('Lilly') stimulating pulses necessary in a neurological prosthesis? I historical background; Pt resting potential; Q studies. Med Biol Eng Comput 24(1):41-49

3. Lilly JC, Hughes JR (1955) Brief, noninjurious electric waveform for stimulation of the brain. Science 121:468-469

4. Liu X, Demosthenous A, Donaldson N (2008) Five valuable functions of blocking capacitors in stimulators. In: Proceedings of the 13th annual international conference of the FES society (IFESS08), Freiburg, pp 322-324

5. Liu X, Demosthenous A, Donaldson N (2008) An integrated implantable stimulator that is fail-safe without off-chip blockingcapacitors. IEEE Trans Biomed Circuit Syst 2(3):231-244

6. Malmivuo J, Plonsey R (1995) Bioelectromagnetism: principles and applications of bioelectric and biomagnetic fields. Oxford University Press, New York

7. Merrill DR, Bikson M, Jeffreys JGR (2005) Electrical stimulation of excitable tissue: design of efficacious and safe stimulation protocols. J Neurosci Method 141:171-198

8. Nag S, Jia X, Thakor NV, Sharma D (2013) Flexible charge balanced stimulator with $5.6 \mathrm{fC}$ accuracy for $140 \mathrm{nC}$ injections. IEEE Trans Biomed Circuit Syst 7(3):266-275

9. Nonclercq A, Lonys L, Vanhoestenberghe A, Demosthenous A, Donaldson N (2012) Safety of multi-channel stimulation implants: a single blocking capacitor per channel is not sufficient after single-fault failure. Med Biol Eng Comput 50(4):403-410

10. Parramon J, Nimmagadda K, Feldman E, He Y (2013) Multielectrode implantable stimulator device with a single current patch decoupling capacitor. US Patent 8,369,963

11. Prutchi D, Norris M (2005) Chapter 7 in design and development of medical electronic instrumentation: a practical perspective of the design, construction and test of medical devices. Wiley, New Jersey

12. Rose TL, Robblee LS (1990) Electrical stimulation with Pt electrodes VIII electrochemically safe charge injection limits with 0.2 ms pulses. IEEE Trans Biomed Eng 37:1118-1120 
13. Sit J, Sarpeshkar R (2007) A low-power blocking-capacitor-free charge-balanced electrode-stimulator chip with less than $6 \mathrm{nA}$ DC error for 1-mA full-scale stimulation. IEEE Trans Biomed Circuit Syst 1(3):172-183

14. Sooksood K, Stieglitz T, Ortmanns M (2009) An experimental study on passive charge balancing. Adv Radio Sci 7:197-200
15. Sooksood K, Stieglitz T, Ortmanns M (2010) An active approach for charge balancing in functional electrical stimulation. IEEE Trans Biomed Circuit Syst 4:162-170

16. St. Jude Medical Inc (2014) Eon Rechargeable IPG tech specs. http://professional.sjm.com/products/neuro/scs/generators/ eon-rechargeable-ipg 